\title{
COMPUTING PERIOD INTEGRALS OF RIGID DOUBLE OCTIC CALABI-YAU THREEFOLDS WITH PICARD-FUCHS OPERATOR
}

\author{
TYMOTEUSZ CHMIEL
}

\begin{abstract}
We present a method for numerical computation of period integrals of a rigid Calabi-Yau threefold using Picard-Fuchs operator of a one-parameter smoothing. Our method gives a possibility of computing the lattice of period integrals of a rigid double octic without any explicit knowledge of its geometric properties, employing only simple facts from the theory of Fuchsian equations and computations in MAPLE with a library for differential equations. As a surprising consequence we also get approximations of additional integrals related to a singular (nodal) model of the considered Calabi-Yau threefold.
\end{abstract}

\section{INTRODUCTION}

In this paper by a double octic we understand a Calabi-Yau threefold obtained as a resolution of singularities of a double cover of $\mathbb{P}^{3}$ branched along a sum of eight planes in specific configurations. There exist eleven rigid double octic Calabi-Yau threefolds defined over $\mathbb{Q}$; they are modular with explicitly given modular form (for details see [8]).

If $X$ is a rigid Calabi-Yau threefold, then for a fixed complex volume form $\omega \in H^{3,0}(X)$ period integrals form a lattice

$$
\Lambda:=\left\{\int_{\gamma} \omega: \gamma \in H_{3}(X, \mathbb{Z})\right\} \subset \mathbb{C} .
$$

Cynk and van Straten 2] used an explicit numerical integration over three-cycles defined by real polyhedral cells to compute approximations of period integrals of eleven rigid double octic Calabi-Yau threefolds. These computations gave numerical evidences that the period integrals are proportional to the special values of the $L$-function for the corresponding modular form.

We start with the observation that birational models of double octics appear as singular elements at conifold points of one-parameter families of manifolds $\left\{X_{t}\right\}_{t \in \mathbb{C}}$, whose general element is a Calabi-Yau threefold with $h^{1,2}(X)=1$. With each of these families we can associate a holomorphic solution of certain differential equation of Fuchsian type.

Using basic properties of the monodromy group of a Fuchsian equation, we were also able to obtain values proportional to the special values of $L$-functions of respective modular forms by means of solutions' analytic continuation along a loop starting near the conifold point and encircling the point of maximal unipotent monodromy.

Our method gives a possibility of computing the lattice of period integrals of a rigid double octic without any explicit knowledge of its geometric properties, employing only simple facts from the theory of Fuchsian equations and computations in MAPLE with a library for differential equations. As a surprising consequence we also get approximations of additional integrals related to a singular (nodal) model of considered CalabiYau threefold.

Recently Ruddat and Siebert proposed a different method of computing period integrals using toric degenerations (see [9]).

The first two sections of our paper are of preliminary nature - we collect necessary information on double octic Calabi-Yau threefolds and Picard-Fuchs operator. Third section contains the description of our method for computing period integrals, and in the fourth section we present the implementation in MAPLE. The last section collects the results of our computation. In the Appendix, for the reader's convenience, we list the

2010 Mathematics Subject Classification. Primary: 14J32; Secondary: 11F67, 14D05.

Key words and phrases. Calabi-Yau threefolds, modular forms, period integrals, Picard-Fuchs operator. 
Picard-Fuchs operators of one-parameter families of double octic Calabi-Yau threefolds with a MUM point and a conifold point.

This paper is an extended version of my bachelor thesis written at the Jagiellonian University under the supervision of prof. Sławomir Cynk.

\section{Double octic Calabi-Yau threefolds}

In this paper we shall be concerned with Calabi-Yau threefolds constructed as crepant resolutions of double covers of the projective space $\mathbb{P}^{3}$ branched along an arrangement of eight planes $S=P_{1} \cup \cdots \cup P_{8}$. If the arrangement has no sixfold point and no fourfold line, the double cover admits a projective crepant resolution of singularities; we call the resulting Calabi-Yau threefold a double octic.

A Calabi-Yau threefold $X$ is called rigid if it admits no infinitesimal deformations or equivalently if $h^{1,2}(X)=0$. C. Meyer in [8] gave a list of eleven rigid double octic Calabi-Yau threefolds defined over $\mathbb{Q}$ and 63 examples of one-parameter families; the numbering of arrangements in our paper follows the one given there.

In tab. 1 we give the equations of eleven arrangements of eight planes which result in a rigid resolution. For details on double octics we refer the reader to the monograph [8].

\begin{tabular}{|c|c|}
\hline Arrangement & Equation \\
\hline 1 & $x y z u(x+y)(y+z)(z+u)(u+x)$ \\
\hline 3 & $x y z u(x+y)(y+z)(y-u)(x-y-z+u)$ \\
\hline 19 & $x y z u(x+y)(y+z)(x-z-u)(x+y+z-u)$ \\
\hline 32 & $x y z u(x+y)(y+z)(x-y-z-u)(x+y-z+u)$ \\
\hline 69 & $x y z u(x+y)(x-y+z)(x-y-u)(x+y-z-u)$ \\
\hline 93 & $x y z u(x+y)(x-y+z)(y-z-u)(x+z-u)$ \\
\hline 238 & $x y z u(x+y+z-u)(x+y-z+u)(x-y+z+u)(-x+y+z+u)$ \\
\hline 239 & $x y z u(x+y+z)(x+y+u)(x+z+u)(y+z+u)$ \\
\hline 240 & $x y z u(x+y+z)(x+y-z+u)(x-y+z+u)(x-y-z-u)$ \\
\hline 241 & $x y z u(x+y+z+u)(x+y-z-u)(y-z+u)(x+z-u)$ \\
\hline 245 & $x y z u(x+y+z)(y+z+u)(x-y-u)(x-y+z+u)$ \\
\hline
\end{tabular}

TABLE 1. Rigid double octic Calabi-Yau threefolds

By the modularity theorem (cf. [5]) every rigid Calabi-Yau threefold defined over $\mathbb{Q}$ is modular, i.e. its $L$-series is equal to an $L$-series $L(f, s)$ of a modular form $f$ of weight four for some $\Gamma_{0}(N)$. In the table below we give the modular forms corresponding to rigid arrangements (we again adopt the notation from [8]):

\begin{tabular}{|r|l|l|}
\hline Form & q-series expansion & Arrangements \\
\hline $6 / 1$ & $q-2 q^{2}-3 q^{3}+4 q^{4}+6 q^{5}+6 q^{6}-16 q^{7}+O\left(q^{8}\right)$ & 240,245 \\
$8 / 1$ & $q-4 q^{3}-2 q^{5}+24 q^{7}-11 q^{9}-44 q^{11}+O\left(q^{12}\right)$ & $1,32,69,93,238,241$ \\
$12 / 1$ & $q+3 q^{3}-18 q^{5}+8 q^{7}+9 q^{9}+36 q^{11}+O\left(q^{12}\right)$ & 239 \\
$32 / 1$ & $q+22 q^{5}-27 q^{9}+O\left(q^{12}\right)$ & 19 \\
$32 / 2$ & $q+8 q^{3}-10 q^{5}+16 q^{7}+37 q^{9}-40 q^{11}+O\left(q^{12}\right)$ & 3 \\
\hline
\end{tabular}

TABLE 2. Modular forms for rigid double octics

For our purposes it is crucial that every rigid double octic is birational to a special member $X_{t_{0}}$ of a oneparameter family $X_{t}$; all special elements of one-parameter families of double octics are given in [1]. Taking the resolution of the general member of a family of branched double covers, we get a partial resolution of the special element corresponding to a rigid arrangement. This gives a special form of geometric transition. 


\section{PicARd-Fuchs operator of a ONe-Parameter FAMily}

Let $X$ be a Calabi-Yau manifold with $h^{1,2}(X)=1$. By the Bogomolov-Tian-Todorov theorem $X$ has a one-dimensional deformation space $\left\{X_{t}\right\}_{t \in V}$, where $V$ is a neighbourhood of 0 in $\mathbb{C}$ and $X_{0}=X$. The family $\left\{X_{t}\right\}_{t \in V}$ is locally trivial, i.e. it is locally diffeomorphic to $X_{0} \times \Delta$.

Let $\omega_{t}$ be the complex volume form on $X_{t}$, depending holomorphically on $t$. If we fix a 3-cycle $\gamma_{0} \in H_{3}\left(X_{0}, \mathbb{Z}\right)$ on $X_{0}$, by local triviality we can extend it to $\gamma_{t} \in H_{3}\left(X_{t}, \mathbb{Z}\right)$ for all $t \in V$. The function $y(t):=\int_{\gamma_{t}} \omega_{t}$ is called the period function.

Period function of a one-parameter family of Calabi-Yau threefolds over a complement of a finite set in $\mathbb{P}^{1}$ satisfies a differential equation, called the Picard-Fuchs equation of this family. It is a linear differential equation of order four

$$
y^{(4)}+p_{1} y^{(3)}+p_{2} y^{\prime \prime}+p_{3} y^{\prime}+p_{4} y=0,
$$

with rational coefficients $p_{i} \in \mathbb{C}(X)$. We can also write the Picard-Fuchs operator in the form

$$
\Theta^{4}+q_{1} \Theta^{3}+q_{2} \Theta^{2}+q_{3} \Theta+q_{4}=0,
$$

where $\Theta=t \cdot \frac{d}{d t}$ denotes the logarithmic derivation, $q_{i} \in \mathbb{C}(X)$.

The Picard-Fuchs operator of a one-parameter family of Calabi-Yau threefolds is Fuchsian, i.e. it has only regular singular points. Equivalently the functions $q_{i}$ are in fact polynomials. More detailed information on Picard-Fuchs operators of one-parameter families of Calabi-Yau threefolds can be found in [10].

The local exponents of the Picard-Fuchs operator at a singular point $t_{0}$ corresponding to a singular CalabiYau threefold are equal to $\{\alpha-\delta, \alpha, \alpha, \alpha+\delta\}$ with rational $\alpha, \delta, \delta>0$. Such a regular singular point $t_{0}$ is called a conifold point (or a $C$ point). After a pullback we can assume the local exponents to be $\{0,1,1,2\}$ and then there is a fundamental system of solutions of the Picard-Fuchs equation of the form

$$
f_{1}, f_{2}, f_{3}+\log \left(t-t_{0}\right) f_{2}, f_{4}
$$

where for $i=1, \ldots, 4$ the functions $f_{i}$ are holomorphic in the neighbourhood of the conifold point and $f_{1}, f_{2}, f_{3}$, and $f_{4}$ have orders $0,1,1$, and 2 respectively.

Our computation depends also on the existence of a point of maximal unipotent monodromy (MUM) regular singular point $t_{0}$ with local exponents $\{0,0,0,0\}$. At a MUM point we can choose a fundamental system of solutions of the form

$$
\begin{gathered}
f_{1}, f_{2}+\log \left(t-t_{0}\right) f_{1}, f_{3}+\log \left(t-t_{0}\right) f_{2}+\frac{1}{2}\left(\log \left(t-t_{0}\right)\right)^{2} f_{1}, \\
f_{4}+\log \left(t-t_{0}\right) f_{3}+\frac{1}{2}\left(\log \left(t-t_{0}\right)\right)^{2} f_{2}+\frac{1}{6}\left(\log \left(t-t_{0}\right)\right)^{3} f_{1},
\end{gathered}
$$

where for $i=1, \ldots, 4$ the functions $f_{i}$ are holomorphic in its neighbourhood and $f_{1}$ has a non-zero free term.

We shall also use notation $M U M+a$ point, resp. $C+a$ point, $a \in \mathbb{Q}$, for a point with local exponents $\{a, a, a, a\}$, resp. $\{a, a+1, a+1, a+2\}$, and an appropriate fundamental system of solutions. Similarly, $a C$ point denotes a point with local exponents $\{0, a, a, 2 a\}$.

A solution $f$ of the Picard-Fuchs operator at point $t_{0}$ can be continued along any path $\gamma:[0,1] \longrightarrow \mathbb{P}^{1} \backslash \Sigma$ with $\gamma(0)=t_{0}$, omitting the set of singular points $\Sigma$, and produces a solution $T_{\gamma}(f)$ at the point $\gamma(1)$. Moreover, the continuations $T_{\gamma}(f)$ along homotopic paths are equal.

Continuation along a loop based at any point $t \notin \Sigma$ gives a linear transformation of the space of solutions in a neighbourhood of this point. If $t_{0} \in \Sigma$ is a singular point and we take a small disc $\Delta$ centered at $t_{0}$ that does not contain any other singularities, the continuation along the boundary of $\Delta$ defines the local monodromy operator $T_{t_{0}}$.

\section{Description OF THE METHOD}

We shall consider a family $X_{t}$ of double octic Calabi-Yau threefolds such that a general member of this family satisfies $h^{1,2}\left(X_{t}\right)=1, t_{0}$ is a conifold point and there is a MUM point, which we may assume to be 0 . Moreover assume that $X_{t_{0}}$ is a singular variety that resolves to a rigid Calabi-Yau threefold $\bar{X}_{t_{0}}$. 
The computations in 2] yield numerical evidences that real and imaginary period integrals of $\bar{X}_{t_{0}}$ are integral multiples of $\pi^{2} L(f, 1)$ and $\pi L(f, 2)$, where $L(f, 1)$ and $L(f, 2)$ are the special values of the $L$-function of the modular form corresponding to $\bar{X}_{t_{0}}$.

We shall present a method which in principle allows one to compute an arbitrarily precise numerical approximation of periods of $\bar{X}_{t_{0}}$. In our computations we shall not use any geometric properties of the one-parameter family, in particular we shall not use the fact that $X_{t}$ is a double octic. In fact, we will only use the Picard-Fuchs operator of the family $X_{t}$ computed in 3 . Due to this fact, we have refrained from listing the equations of one-parameter families and in the Appendix we present only their associated Picard-Fuchs operators.

Let us recall that in our situation the element of the family of Calabi-Yau threefolds $X_{t}$ is defined by a resolution of a double covering of $\mathbb{P}^{3}$ branched along an arrangement of eight planes $S_{t}=P_{1}^{t} \cup \cdots \cup P_{8}^{t}$. Special elements $X_{t_{0}}$ of this family correspond to a choice of four indices $1 \leq i_{1}<i_{2}<i_{3}<i_{4} \leq 8$ such that the planes $P_{i_{1}}^{t}, P_{i_{2}}^{t}, P_{i_{3}}^{t}, P_{i_{4}}^{t}$ are in general position for generic $t$, but $P_{i_{1}}^{t_{0}}, P_{i_{2}}^{t_{0}}, P_{i_{3}}^{t_{0}}, P_{i_{4}}^{t_{0}}$ intersect. The resolution of singularities of a generic element of a family of double covers yields also a partial resolution of the double cover at $t_{0}$.

Assume that the intersection of the planes $P_{i_{1}}^{t_{0}}, P_{i_{2}}^{t_{0}}, P_{i_{3}}^{t_{0}}, P_{i_{4}}^{t_{0}}$ is a fourfold point of $S_{t_{0}}$ which is not contained in a triple line of $S_{t_{0}}$ (we call such a singular point $p_{4}^{0}$ ). Then the degeneration of $S_{t}$ at $t_{0}$ is given by a vanishing tetrahedron, which gives a vanishing cycle in $X_{t}$. The singular element $X_{t_{0}}$ has two ordinary double points (nodes) as its only singularities.

If the special fiber is nodal, gluing a 4-cell along the vanishing cycle we get topological space homotopic to the special fiber. The same holds true for a small resolution with a 3-cell glued along the exceptional line.

Theorem 1 ([11, Ch. II]). Let $X_{t}, t \in \Delta$, be a family of projective varieties such that

- $X_{t}$ is a (smooth) Calabi-Yau threefold with $h^{1,2}\left(X_{t}\right)=1$, for $t \neq 0$,

- $X_{0}$ is a nodal variety such that a small resolution $\bar{X}_{0}$ is a rigid Calabi-Yau threefold.

Then $H_{3}\left(X_{0}\right) \cong \mathbb{Z}^{3} \oplus$ torsions.

As an integral over a torsion cycle vanishes, we will only consider homology groups modulo torsions. Denoting by $\bar{X}_{t_{0}}$ a (projective) small resolution of $X_{t_{0}}$ we get

$$
H_{3}\left(X_{t}, \mathbb{Z}\right) \cong \mathbb{Z}^{4}, \quad H_{3}\left(X_{t_{0}}, \mathbb{Z}\right) \cong \mathbb{Z}^{3}, \quad H_{3}\left(\bar{X}_{t_{0}}, \mathbb{Z}\right) \cong \mathbb{Z}^{2}
$$

The group $H_{3}\left(X_{t}, \mathbb{Z}\right)$ is spanned by $H_{3}\left(X_{t_{0}}, \mathbb{Z}\right)$ and the vanishing cycle. From considerations in $[2]$ it follows that the map

$$
H_{3}\left(\bar{X}_{t_{0}}, \mathbb{Z}\right) \longrightarrow H_{3}\left(X_{t_{0}} \mathbb{Z}\right)
$$

is injective. Moreover, a polyhedral 3-cycle belongs to the image of this map iff it is locally symmetric at the $p_{4}^{0}$ point. Consequently, the integrals of the 3 -form

$$
\omega_{t_{0}}:=\frac{d x \wedge d y \wedge d z}{\sqrt{F_{t_{0}}(x, y, z)}},
$$

where $F_{t_{0}}(x, y, z)$ is an equation of the octic arrangement $S_{t_{0}}$ in an affine chart, over polyhedral 3-cycles in $X_{t_{0}}$ generate a group of rank at most 3 in $\mathbb{C}$, while integrals over polyhedral 3-cycles in $H_{3}\left(\bar{X}_{t_{0}}\right)$ generate a rank two subgroup (commensurable with the full periods lattice of $\bar{X}_{t_{0}}$ ).

Since we will consider only those integrals the computations in 2] take into account, in the table below we list the generators of integrals over polyhedrals 3-cycles on a singular double cover of a rigid arrangement (since we impose no symmetry condition on the cycles at the $p_{4}^{0}$ point, we can get more than three independent integrals): 


\begin{tabular}{r|rr|rr} 
Arr. & Real integrals & & Imaginary integrals \\
\hline 3 & 14.303841078 & 18.695683053 & $41.413458745 \mathrm{i}$ & \\
19 & 12.3280533145 & 19.3301891966 & $12.3280533145 \mathrm{i}$ & $19.3301891966 \mathrm{i}$ \\
32 & 11.13352966 & 16.85672240 & $17.34237466 \mathrm{i}$ & \\
69 & 11.13352966 & 16.85672240 & $17.34237465 \mathrm{i}$ & \\
93 & 8.42836120319 & 11.1335296603 & $17.3423746625 \mathrm{i}$ & \\
239 & 13.1823084825 & 17.6714531944 & $11.7425210928 \mathrm{i}$ & \\
240 & 3.99263311132 & 6.94406875218 & $4.80390756451 \mathrm{i}$ & $6.9176905115 \mathrm{i}$ \\
245 & 3.99263311132 & 6.94406875217 & $5.38024923409 \mathrm{i}$ & $7.49403218155 \mathrm{i}$
\end{tabular}

The integrals over polyhedral 3-cycles in $H_{3}\left(X_{t_{0}}\right)$ form a subgroup of rank at most 3 in the group generated by the entries of the above table corresponding to $\bar{X}_{t_{0}}$. Note that the group $H_{3}\left(X_{t_{0}}\right)$ depends not only on the rigid arrangement but also on the choice of the one-parameter family.

Now recall that at the conifold point $t_{0}$ we have a fundamental system of solutions of the form

$$
f_{1}, f_{2}, f_{3}+\log \left(t-t_{0}\right) f_{2}, f_{4} .
$$

Up to multiplication by a constant, two elements of this basis are uniquely determined: $f_{4}$ as the unique element of order 2 and $f_{2}$ as the coefficient of the logarithmic term.

For any solution $f$ of the differential equation near a conifold point $t_{0}$, the local monodromy around $t_{0}$ is of the form $T_{t_{0}}(f)=f+c \cdot f_{2}$, where $c$ is a constant. The solution $f_{2}$ is therefore proportional to $N_{t_{0}} f$, where $N_{t_{0}}=T_{t_{0}}-$ Id. In our case where $X_{t_{0}}$ is a nodal variety, $f_{2}(t)$ equals the integral over the vanishing cycle on $X_{t}$.

Now for a differential operator $\mathcal{P}$ with a MUM point at 0 and a conifold point $t_{0}$ denote by $\mathcal{L}_{\mathcal{P}, t_{0}}$ the additive group generated by $\left\{\operatorname{Re}\left(T_{0}^{n}\left(f_{2}\right)\right), \operatorname{Im}\left(T_{0}^{n}\left(f_{2}\right)\right): n \in \mathbb{N}\right\}$. Observe that in our case this will be a subgroup of integrals over cycles in $H_{3}\left(X_{t_{0}}\right)$ and therefore will have rank at most 3.

Assume that we have two different Picard-Fuchs operators $\mathcal{P}$ and $\mathcal{P}^{\prime}$ with conifold points $t_{0}$ and $t_{0}^{\prime}$ such that $\bar{X}_{t_{0}} \cong \bar{X}_{t_{0}^{\prime}}$. It follows from the considerations above that if $\mathcal{L}_{\mathcal{P}, t_{0}}$ and $\mathcal{L}_{\mathcal{P}^{\prime}, t_{0}^{\prime}}$ are of rank 3 and for some $\alpha \in \mathbb{C}$ the intersection $\mathcal{L}_{\mathcal{P}, t_{0}} \cap \alpha \mathcal{L}_{\mathcal{P}^{\prime}, t_{0}^{\prime}}$ is a lattice, this lattice is commensurable with the lattice of period integrals of $\bar{X}_{t_{0}}$.

On the other hand, in all considered cases if $\mathcal{L}_{\mathcal{P}, t_{0}}$ already is a lattice, it is (numerically) commensurable with the lattice of period integrals.

\section{Computing the PERIOds}

We now describe the implementation of the above general idea. Using MAPLE's procedure formal_sol we are able to determine the solution $f_{2}$ in a neighbourhood of the conifold point $t_{0}$. We then choose a polygon chain $L=\left(a_{0}, \ldots, a_{n+1}\right)$ such that $a_{0}=a_{n+1}=t_{0}+\epsilon,|\epsilon| \ll 1$, and the winding number of $L$ around every singular point of the equation - except for the MUM point at 0 - equals 0 and the winding number around 0 equals 1.

Let $g_{0}, g_{1}, g_{2}, g_{3}$ be a fundamental system of solutions at the point $a_{i+1}$, again computed with the MAPLE's formal_sol procedure. Note that in every point of the curve $L$ we have a fundamental system of solutions of orders $0,1,2,3$. Therefore given a solution $g$ in a neighbourhood of $a_{i}$ whose disc of convergence has non-empty intersection with the discs of convergence of all solutions at $a_{i+1}$, we can fix a point $c_{i}$ in this intersection and consider the system of equations

$$
\left[\begin{array}{c}
g\left(c_{i}\right) \\
g^{\prime}\left(c_{i}\right) \\
g^{\prime \prime}\left(c_{i}\right) \\
g^{\prime \prime \prime}\left(c_{i}\right)
\end{array}\right]=\left[\begin{array}{c}
X_{1} g_{0}\left(c_{i}\right)+X_{2} g_{1}\left(c_{i}\right)+X_{3} g_{2}\left(c_{i}\right)+X_{4} g_{3}\left(c_{i}\right) \\
X_{1} g_{0}^{\prime}\left(c_{i}\right)+X_{2} g_{1}^{\prime}\left(c_{i}\right)+X_{3} g_{2}^{\prime}\left(c_{i}\right)+X_{4} g_{3}^{\prime}\left(c_{i}\right) \\
X_{1} g_{0}^{\prime \prime}\left(c_{i}\right)+X_{2} g_{1}\left(c_{i}\right)+X_{3} g_{2}^{\prime \prime}\left(c_{i}\right)+X_{4} g_{3}^{\prime \prime}\left(c_{i}\right) \\
X_{1} g_{0}^{\prime \prime \prime}\left(c_{i}\right)+X_{2} g_{1}^{\prime \prime \prime}\left(c_{i}\right)+X_{3} g_{2}^{\prime \prime \prime}\left(c_{i}\right)+X_{4} g_{3}^{\prime \prime \prime}\left(c_{i}\right)
\end{array}\right] .
$$

This gives us representation of $g$ in the basis $g_{0}, g_{1}, g_{2}, g_{3}$ and we can continue it analytically to the point $a_{i+1}$ (cf. [4). 
In our application of this method, we begin with the solution $f_{2}$ at a point close to the conifold point $t_{0}$. Repeating the aforementioned computation for all vertices of $L$, we continue the solution $f_{2}$ around the point 0 and obtain a new solution $\tilde{f}_{2}$ at $t_{0}+\epsilon$. In our computations we always took $c_{i}=\frac{1}{2}\left(a_{i}+a_{i+1}\right)$, therefore while choosing vertices of the polyline it was important to check that $c_{i}$ is sufficiently close to the centers of the discs of convergence at $a_{i}$ and $a_{i+1}$ to avoid potential problems with the rate of convergence.

When this procedure is completed, we end up with a new solution $\tilde{f}_{2}:=T_{0}\left(f_{2}\right)$. As our goal is to determine $\mathcal{L}_{\mathcal{P}, t_{0}}$, we repeat the procedure now starting with $\tilde{f}_{2}$ instead of $f_{2}$, getting a new solution $\tilde{\tilde{f}}_{2}$ and so on. Then we use the following:

Theorem 2. Let $W_{n}:=T_{0}^{n}\left(f_{2}\right)\left(t_{0}\right), n \in \mathbb{N}$. The sequence satisfies the following recursion

$$
W_{n+4}=4 W_{n+3}-6 W_{n+2}+4 W_{n+1}-W_{n} .
$$

Proof. The monodromy operator $T_{0}$ around a MUM point has a single Jordan block with eigenvalue 1:

$$
\left(\begin{array}{llll}
1 & 1 & 0 & 0 \\
0 & 1 & 1 & 0 \\
0 & 0 & 1 & 1 \\
0 & 0 & 0 & 1
\end{array}\right) .
$$

Consequently the characteristic polynomial of $T_{0}$ equals $\chi(T)=(T-1)^{4}$. By the Cayley-Hamilton theorem

$$
T_{0}^{4}-4 T_{0}^{3}+6 T_{0}^{2}-4 T_{0}+6 \mathrm{Id}=0
$$

and the theorem follows.

We therefore see that all possible values of $\operatorname{Re}\left(W_{n}\right)$ and $\operatorname{Im}\left(W_{n}\right)$ are integral linear combinations of the first four and the generators for those values will generate the entire $\mathcal{L}_{\mathcal{P}, t_{0}}$.

Additional difficulty was posed by examples where instead of MUM and C points we encountered points of type MUM $+a, \mathrm{C}+a$ or $\frac{1}{m} \mathrm{C}$ for some $a \in \mathbb{Q}$ and $m \in \mathbb{N}$. In two former cases it is relatively easy to change the local exponents such that we get the desired basis: indeed, to change local exponents at 0 one only needs to represent the differential operator using the logarithmic derivative $\Theta$ and then substitute $\Theta \mapsto \Theta+a$. However normalizing basis at $\frac{1}{m} \mathrm{C}$ points would require us to use the substitution $\left(t-t_{0}\right) \mapsto\left(t-t_{0}\right)^{m}$, producing new regular singular points in the process and making it harder to find the correct polyline. Because of this inconvenience, we have decided to perform the computations for this type of singular points using their natural basis $\left(t-t_{0}\right)^{\frac{1}{m}} f_{1},\left(t-t_{0}\right)^{\frac{1}{m}} f_{2},\left(t-t_{0}\right)^{\frac{1}{m}}\left(f_{3}+\log \left(t-t_{0}\right) f_{2}\right),\left(t-t_{0}\right)^{\frac{1}{m}} f_{4}$ and take $\left(t-t_{0}\right)^{\frac{1}{m}} f_{2}$ as the initial solution.

Example. We shall now present the details of computations in the simplest case of Arrangement 2. It has the equation

$$
(y+z)(x+y)(z+u)(t u+x) x y z u,
$$

where $t$ is the parameter and $x, y, z, u$ are coordinates in $\mathbb{P}^{3}$. Picard-Fuchs operator for this family is

$$
\mathcal{P}_{2}:=\Theta^{4}-\frac{1}{16} t(2 \Theta+1)^{4} .
$$

The equation $\mathcal{P}_{2}=0$ has three regular singular points; we present them in the following table, called the Riemann scheme, together with their local exponents:

$$
\left\{\begin{array}{ccc}
0 & 1 & \infty \\
\hline 0 & 0 & 1 / 2 \\
0 & 1 & 1 / 2 \\
0 & 1 & 1 / 2 \\
0 & 2 & 1 / 2
\end{array}\right\}
$$

Since 1 is a conifold point, 0 is a MUM point and the third regular singular point is located at infinity, we take

$$
\left(1, \frac{3}{4}, \frac{1}{2}, \frac{1}{4}, \frac{1}{4}+\frac{i}{4}, \frac{i}{4},-\frac{1}{4}+\frac{i}{4},-\frac{1}{4},-\frac{1}{4}-\frac{i}{4},-\frac{i}{4}, \frac{1}{4}-\frac{i}{4}, \frac{1}{4}, \frac{1}{2}, \frac{3}{4}, 1\right)
$$

as the vertices of the polyline $L$. 
Working with the precision Digits : $=30$ and with the parameter 'order' $=100$ for the procedure formal_sol from the DEtools library, we continue analytically the solution

$$
\begin{aligned}
f_{2}=(t-1)+\frac{3}{4}(t-1)^{2}-\frac{29}{48}(t-1)^{3}+\frac{49}{96}(t-1)^{4}-\frac{2277}{5120}(t-1)^{5}+\frac{8107}{20480}(t-1)^{6} \\
\\
\quad-\frac{205223}{573440}(t-1)^{7}+\frac{37551}{114688}(t-1)^{8}-\frac{11413801}{37748736}(t-1)^{9}+\ldots
\end{aligned}
$$

along $L$. After the first encircling we got

$$
\tilde{f}_{2}(1)=-11.3440218793908710004979185926+1.93350327192382796832769889845 \cdot 10^{-23} i,
$$

after the second -

$$
\widetilde{\tilde{f}}_{2}(1)=-45.3760875175634840019915624157+28.1143988476259022087394753013 i,
$$

and so forth.

It is then checked that the sequences

$$
\frac{\operatorname{Re}\left(T_{0}^{n}\left(f_{2}\right)(1)\right)}{\operatorname{Re}\left(T_{0}\left(f_{2}\right)(1)\right)} \text { and } \frac{\operatorname{Im}\left(T_{0}^{n}\left(f_{2}\right)(1)\right)}{\operatorname{Im}\left(T_{0}^{2}\left(f_{2}\right)(1)\right)}
$$

for $n=1,2,3,4$ within precision of 20 digits read $(1,4,9,16)$ and $(0,1,4,10)$. Using Theorem 2 we see that in this case $\mathcal{L}_{\mathcal{P}_{2}, t_{0}}$ is already a lattice.

The resolution of singularities at the considered conifold point corresponds to the double octic of Arrangement 1 with the corresponding modular form 8/1. Its special $L$-values are

$$
\begin{aligned}
& L(f, 1) \approx 0.35450068373096471876555989149, \\
& L(f, 2) \approx 0.69003116312339752511910542021 .
\end{aligned}
$$

We can sum up our computations by

$$
\begin{aligned}
& \frac{\operatorname{Re}\left(\tilde{f}_{2}(1)\right)}{L(f, 1)} \approx-32.0000000000000000000000056066, \\
& \pi \cdot \frac{\operatorname{Im}\left(\widetilde{\tilde{f}}_{2}(1)\right)}{L(f, 2)} \approx 127.999999999999999999997974854 .
\end{aligned}
$$

\section{Results of COMPUtation}

In total we have studied 49 conifold points appearing in 29 families of double octics.

In 40 cases the described method suggests that $\mathcal{L}_{\mathcal{P}, t_{0}}$ is already a lattice commensurable with the period lattice of the corresponding rigid double octic. Similarly to the example above, in fact we get not only the commensurability of lattices but it also appears that the generators computed by the means of our method are multiples of $L(f, 1)$ and $\frac{L(f, 2)}{2 \pi i}$. In some cases they are multiples of $\sqrt{2} L(f, 1)$ and $\sqrt{2} \frac{L(f, 2)}{2 \pi i}$; the reason is that in order to get the twist of the modular form with the smallest possible level, the equation of the corresponding arrangement of planes was multiplied by 2 (cf. [2]).

The following table presents the generators in those 40 cases:

\begin{tabular}{|c|c|c|c|c|c|}
\hline Arr. & $\begin{array}{l}\text { confiold } \\
\text { point }\end{array}$ & $\begin{array}{l}\text { rigid } \\
\text { Arr. }\end{array}$ & $\begin{array}{l}\text { modular } \\
\text { form }\end{array}$ & real period & imaginary period \\
\hline 2 & 1 & 1 & $8 / 1$ & $32 L(f, 1)$ & $256 \frac{L(f, 2)}{2 \pi i}$ \\
\hline 8 & -1 & 1 & $8 / 1$ & $64 i \frac{L(f, 2)}{2 \pi i}$ & $64 i L(f, 1)$ \\
\hline 10 & 0 & 1 & $8 / 1$ & $64 i \frac{L(f, 2)}{2 \pi i}$ & $32 i L(f, 1)$ \\
\hline 10 & -1 & 1 & $8 / 1$ & $32 L(f, 1)$ & $64 \frac{L(f, 2)}{2 \pi i}$ \\
\hline 16 & -1 & 1 & $8 / 1$ & $32 L(f, 1)$ & $64 \frac{L(f, 2)}{2 \pi i}$ \\
\hline 20 & 0 & 3 & $32 / 2$ & $4 L(f, 1)$ & $16 \frac{L(f, 2)}{2 \pi i}$ \\
\hline 20 & -1 & 1 & $8 / 1$ & $64 i \frac{L(f, 2)}{2 \pi i}$ & $96 i L(f, 1)$ \\
\hline
\end{tabular}




\begin{tabular}{|c|c|c|c|c|c|}
\hline 36 & -1 & 32 & $8 / 1$ & $128 i \frac{L(f, 2)}{2 \pi i}$ & $128 i L(f, 1)$ \\
\hline 73 & $-\frac{1}{2}$ & 69 & $8 / 1$ & $32 i \frac{L(f, 2)}{2 \pi i}$ & $32 i L(f, 1)$ \\
\hline 94 & -1 & 1 & $8 / 1$ & $16 L(f, 1)$ & $128 \frac{L(f, 2)}{2 \pi i}$ \\
\hline 95 & $\infty$ & 3 & $32 / 2$ & $16 i \frac{L(f, 2)}{2 \pi i}$ & $4 i L(f, 1)$ \\
\hline 99 & $-\frac{1}{2}$ & 19 & $32 / 1$ & $2 \sqrt{2} L(f, 1)$ & $64 \sqrt{2} \frac{L(f, 2)}{2 \pi i}$ \\
\hline 99 & $\infty$ & 19 & $32 / 1$ & $4 \sqrt{2} L(f, 1)$ & $32 \sqrt{2} \frac{L(f, 2)}{2 \pi i}$ \\
\hline 144 & $-\frac{1}{2}$ & 19 & $32 / 1$ & $2 \sqrt{2} L(f, 1)$ & $64 \sqrt{2} \frac{L(f, 2)}{2 \pi i}$ \\
\hline 154 & 0 & 1 & $8 / 1$ & $32 L(f, 1)$ & $64 \frac{L(f, 2)}{2 \pi i}$ \\
\hline 154 & $-\frac{1}{2}$ & 32 & $8 / 1$ & $16 L(f, 1)$ & $32 \frac{L(f, 2)}{2 \pi i}$ \\
\hline 199 & 0 & 1 & $8 / 1$ & $64 i \frac{L(f, 2)}{2 \pi i}$ & $32 i L(f, 1)$ \\
\hline 199 & -1 & 69 & $8 / 1$ & $64 i \frac{L(f, 2)}{2 \pi i}$ & $32 i L(f, 1)$ \\
\hline 242 & $-\frac{1}{2}$ & 238 & $8 / 1$ & $16 L(f, 1)$ & $32 \frac{L(f, 2)}{2 \pi i}$ \\
\hline 242 & $\infty$ & 238 & $8 / 1$ & $64 L(f, 1)$ & $128 \frac{L(f, 2)}{2 \pi i}$ \\
\hline 246 & 0 & 1 & $8 / 1$ & $320 i \frac{L(f, 2)}{2 \pi i}$ & $64 i L(f, 1)$ \\
\hline 246 & $-\frac{1}{2}$ & 241 & $8 / 1$ & $256 i \frac{L(f, 2)}{2 \pi i}$ & $256 i L(f, 1)$ \\
\hline 249 & $-\frac{1}{2}$ & 241 & $8 / 1$ & $128 L(f, 1)$ & $512 \frac{L(f, 2)}{2 \pi i}$ \\
\hline 251 & 0 & 1 & $8 / 1$ & $64 i \frac{L(f, 2)}{2 \pi i}$ & $64 i L(f, 1)$ \\
\hline 251 & $-\frac{1}{2}$ & 93 & $8 / 1$ & $8 \sqrt{2} i \frac{L(f, 2)}{2 \pi i}$ & $16 \sqrt{2} i L(f, 1)$ \\
\hline 251 & $\infty$ & 19 & $32 / 1$ & $4 \sqrt{2} L(f, 1)$ & $16 \sqrt{2} \frac{L(f, 2)}{2 \pi i}$ \\
\hline 253 & 0 & 3 & $32 / 2$ & $8 L(f, 1)$ & $16 \frac{L(f, 2)}{2 \pi i}$ \\
\hline 253 & $-\frac{1}{2}$ & 240 & $6 / 1$ & $5 L(f, 1)$ & $30 \frac{L(f, 2)}{2 \pi i}$ \\
\hline 254 & $-\frac{1}{2}$ & 241 & $8 / 1$ & $8 L(f, 1)$ & $4 \frac{L(f, 2)}{2 \pi i}$ \\
\hline 255 & 2 & 32 & $8 / 1$ & $32 \sqrt{2} i \frac{L(f, 2)}{2 \pi i}$ & $32 \sqrt{2} L(f, 1)$ \\
\hline 256 & -2 & 239 & $12 / 1$ & $32 L(f, 1)$ & $96 \frac{L(f, 2)}{2 \pi i}$ \\
\hline 256 & 2 & 238 & $8 / 1$ & $32 L(f, 1)$ & $256 \frac{L(f, 2)}{2 \pi i}$ \\
\hline 257 & 4 & 240 & $6 / 1$ & $200 L(f, 1)$ & $288 \frac{L(f, 2)}{2 \pi i}$ \\
\hline 259 & 0 & 32 & $8 / 1$ & $32 L(f, 1)$ & $128 \frac{L(f, 2)}{2 \pi i}$ \\
\hline 259 & $\infty$ & 32 & $8 / 1$ & $32 L(f, 1)$ & $128 \frac{L(f, 2)}{2 \pi i}$ \\
\hline 262 & 0 & 1 & $8 / 1$ & $320 i \frac{L(f, 2)}{2 \pi i}$ & $80 L(f, 1)$ \\
\hline 265 & 4 & 69 & $8 / 1$ & $16 L(f, 1)$ & $64 \frac{L(f, 2)}{2 \pi i}$ \\
\hline 268 & $-1 / 2$ & 69 & $8 / 1$ & $160 \sqrt{2} i \frac{L(f, 2)}{2 \pi i}$ & $32 \sqrt{2} i L(f, 1)$ \\
\hline 274 & 0 & 3 & $32 / 2$ & $32 i \frac{L(f, 2)}{2 \pi i}$ & $8 i L(f, 1)$ \\
\hline 274 & $\infty$ & 3 & $32 / 2$ & $32 i \frac{L(f, 2)}{2 \pi i}$ & $8 i L(f, 1)$ \\
\hline
\end{tabular}

Table 3: Results with $\mathcal{L}_{\mathcal{P}, t_{0}}$ of rank 2 
Notice that, except for the Arrangement 245, each double octic corresponding to a rigid arrangement of eight planes appears as a resolution of singularities in at least one conifold point listed above. Therefore for those ten double octics described method seems to allow us to compute period integrals using the PicardFuchs operator of just one one-parameter family.

Now we list the generators for the remaining nine cases:

\begin{tabular}{|c|c|c|c|c|}
\hline \multirow{2}{*}{ Arr. } & \multirow{2}{*}{$\begin{array}{r}\text { special } \\
\text { point }\end{array}$} & \multirow{2}{*}{$\begin{array}{l}\text { rigid } \\
\text { Arr. }\end{array}$} & \multirow{2}{*}{$\begin{array}{c}\text { modular } \\
\text { form }\end{array}$} & real periods \\
\hline & & & & imaginary periods \\
\hline \multirow{3}{*}{5} & \multirow{3}{*}{0} & \multirow{3}{*}{3} & \multirow{3}{*}{$32 / 2$} & 16.7842426348152009451903095600 \\
\hline & & & & $3.78853747194184773010686231258 \mathrm{i}$ \\
\hline & & & & $61.0738884585292464400038239965 \mathrm{i}$ \\
\hline \multirow{3}{*}{5} & \multirow{3}{*}{2} & \multirow{3}{*}{3} & \multirow{3}{*}{$32 / 2$} & 4.19606065870380023629757253576 \\
\hline & & & & $0.94713436798546193252671571987 \mathrm{i}$ \\
\hline & & & & $3.90285969880676841968001329994 \mathrm{i}$ \\
\hline \multirow{3}{*}{20} & \multirow{3}{*}{-2} & \multirow{3}{*}{19} & \multirow{3}{*}{$32 / 1$} & 2.58310412634697457527735648888 \\
\hline & & & & $0.979278824715794481666000593885 \mathrm{i}$ \\
\hline & & & & $4.45674355709313141111341743112 \mathrm{i}$ \\
\hline \multirow{3}{*}{95} & \multirow{3}{*}{$-\frac{1}{2}$} & \multirow{3}{*}{93} & \multirow{3}{*}{$8 / 1$} & 6.21246314816860397127669373665 \\
\hline & & & & $2.99683078705084653614316487029 \mathrm{i}$ \\
\hline & & & & $34.0238543159967756814545903982 \mathrm{i}$ \\
\hline \multirow{3}{*}{244} & \multirow{3}{*}{$\frac{1}{2}$} & \multirow{3}{*}{240} & \multirow{3}{*}{$6 / 1$} & 6.26847094349121003359079636235 \\
\hline & & & & $2.58823590805561845768157001028 \mathrm{i}$ \\
\hline & & & & $32.0498374325403392826453731746 \mathrm{i}$ \\
\hline \multirow{3}{*}{244} & \multirow{3}{*}{2} & \multirow{3}{*}{240} & \multirow{3}{*}{$6 / 1$} & 25.0738837739648401343633473698 \\
\hline & & & & $10.3529436322224738307260280020 \mathrm{i}$ \\
\hline & & & & $128.199349730161357130578977414 \mathrm{i}$ \\
\hline \multirow{3}{*}{253} & \multirow{3}{*}{-2} & \multirow{3}{*}{245} & \multirow{3}{*}{$6 / 1$} & 8.26021099210331426827789806115 \\
\hline & & & & $6.26847094349121003359079492495 \mathrm{i}$ \\
\hline & & & & $7.96011334055139325749281017005 \mathrm{i}$ \\
\hline & & & & 3.13423547174561288123848517920 \\
\hline 274 & $-\frac{1}{2}$ & 245 & $6 / 1$ & $0.839792675513409448977564062085 \mathrm{i}$ \\
\hline & & & & $8.12249522907253404678014309610 \mathrm{i}$ \\
\hline & & & & 5.57197417218209438771001425150 \\
\hline 274 & -2 & 245 & $6 / 1$ & $1.49296475661811062877124575360 \mathrm{i}$ \\
\hline & & & & $14.4399915143798181025349619989 \mathrm{i}$ \\
\hline
\end{tabular}

Table 4: Results with $\mathcal{L}_{\mathcal{P}, t_{0}}$ of rank 3

In each case the real values in $\mathcal{L}_{\mathcal{P}, t_{0}}$ were multiples of the special value, while imaginary parts formed a group of rank 2 .

We see that the remaining case rigid double octic of Arr. 245 appears as a resolution of a special fiber in three cases and we get three rank 3 subgroups: $\mathcal{L}_{\mathcal{P}_{253},-2}, \mathcal{L}_{\mathcal{P}_{274},-\frac{1}{2}}$ and $\mathcal{L}_{\mathcal{P}_{274},-2}$. The intersection $\mathcal{L}_{\mathcal{P}_{253},-2} \cap i \mathcal{L}_{\mathcal{P}_{274},-\frac{1}{2}}$ has rank 2 and is generated by the multiples of the special values: $144 \sqrt{2} i \frac{L(f, 2)}{2 \pi i}$ and $40 \sqrt{2} i L(f, 1)$, as to be expected. 
This means that for all rigid double octics our method allows us to compute the lattice of period integrals with much higher precision than the methods of numerical integration used in 2].

The results in Table 4 can be interpreted by a more detailed analysis of the computations of period integrals carried out in [2] where the period integrals were computed with numerical integration over polyhedral cells. Let us recall the table from the Section 3., listing those integrals:

\begin{tabular}{r|rr|rr} 
Arr. & Real integrals & & Imaginary integrals \\
\hline 3 & 14.303841078 & 18.695683053 & $41.413458745 \mathrm{i}$ & \\
19 & 12.3280533145 & 19.3301891966 & $12.3280533145 \mathrm{i}$ & $19.3301891966 \mathrm{i}$ \\
32 & 11.13352966 & 16.85672240 & $17.34237466 \mathrm{i}$ & \\
69 & 11.13352966 & 16.85672240 & $17.34237465 \mathrm{i}$ & \\
93 & 8.42836120319 & 11.1335296603 & $17.3423746625 \mathrm{i}$ & \\
239 & 13.1823084825 & 17.6714531944 & $11.7425210928 \mathrm{i}$ & \\
240 & 3.99263311132 & 6.94406875218 & $4.80390756451 \mathrm{i}$ & $6.9176905115 \mathrm{i}$ \\
245 & 3.99263311132 & 6.94406875217 & $5.38024923409 \mathrm{i}$ & $7.49403218155 \mathrm{i}$
\end{tabular}

It turns out that we have the following identities, relating unidentified values obtained by means of our method and the generators listed in Table 4

$$
\begin{aligned}
& \pi^{2} \times 3.78853747194184773010686233752 \approx 2 \times 18.695683053 \\
& \pi^{2} \times 61.0738884585292464400038231520 \approx 16 \times 14.303841078+20 \times 18.695683053 \\
& 2 \pi^{2} \times 0.947134367985461932526715642895 \approx 18.695683053 \\
& \pi^{2} \times 3.90285969880676841968003796681 \approx 4 \times 14.303841078-18.695683053 \\
& 2 \pi^{2} \times 0.979278824715794481666000593885 \approx 19.3301891966 \\
& 2 \pi^{2} \times 4.45674355709313141111341743112 \approx 2 \times 12.3280533145-19.3301891966 \\
& \sqrt{2} \pi^{2} \times 2.99683078705084653614316485878 \approx 8.42836120319+3 \times 11.1335296603 \\
& \sqrt{2} \pi^{2} \times 34.023854315996775681454590398 \approx 22 \times 8.42836120319+26 \times 11.1335296603 \\
& \sqrt{2} \pi^{2} \times 2.58823590805561845768157001028 \approx-20 \times 4.80390756451+8 \times 6.9176905115 \\
& \sqrt{2} \pi^{2} \times 32.0498374325403392826453731746 \approx-168 \times 4.80390756451+48 \times 6.9176905115 \\
& \sqrt{2} \pi^{2} \times 10.3529436322224738307263862794 \approx-80 \times 4.80390756451+32 \times 6.9176905115 \\
& \sqrt{2} \pi^{2} \times 128.199349730161357130578977414 \approx-672 \times 4.80390756451+192 \times 6.9176905115 \\
& \sqrt{2} \pi^{2} \times 6.26847094349121003359079492495 \approx 8 \times 3.99263311132+8 \times 6.94406875217 \\
& \sqrt{2} \pi^{2} \times 7.96011334055139325749281017005 \approx 16 \times 6.94406875217 \\
& \sqrt{2} \pi^{2} \times 0.839792675513399595961176504110 \approx-2 \times 5.38024923409+3 \times 7.49403218155 \\
& \sqrt{2} \pi^{2} \times 8.12249522907253404678014309610 \approx-4 \times 5.38024923409+18 \times 7.49403218155 \\
& 9 \sqrt{2} \pi^{2} \times 1.49296475661811062877124579435 \approx-32 \times 5.38024923409+48 \times 7.49403218155 \\
& 9 \sqrt{2} \pi^{2} \times 14.4399915143798181025349622191 \approx-64 \times 5.38024923409+288 \times 7.49403218155
\end{aligned}
$$

Comparing the first two equalities, we get

$$
14.303841078 \approx \frac{\pi^{2}}{16} \cdot(61.0738884585292464400038231520-10 \cdot 3.78853747194184773010686233752) .
$$

In a similar way we can recover much better approximations of other integrals computed in 2]. As a surprising consequence we compute not only the period lattice of a rigid Calabi-Yau threefold but also the periods of the singular model $X_{t_{0}}$ of $\bar{X}_{t_{0}}$. These additional integrals seem to be crucial for better understanding of the transformation matrices between Frobenius basis at a MUM point and a conifold point. 
APPENDIX:

PiCARD-FuChs OPERATORS OF ONE-PARAMETER FAMILIES OF DOUble OCTICS With A MUM POINT AND A CONIFOLD POINT

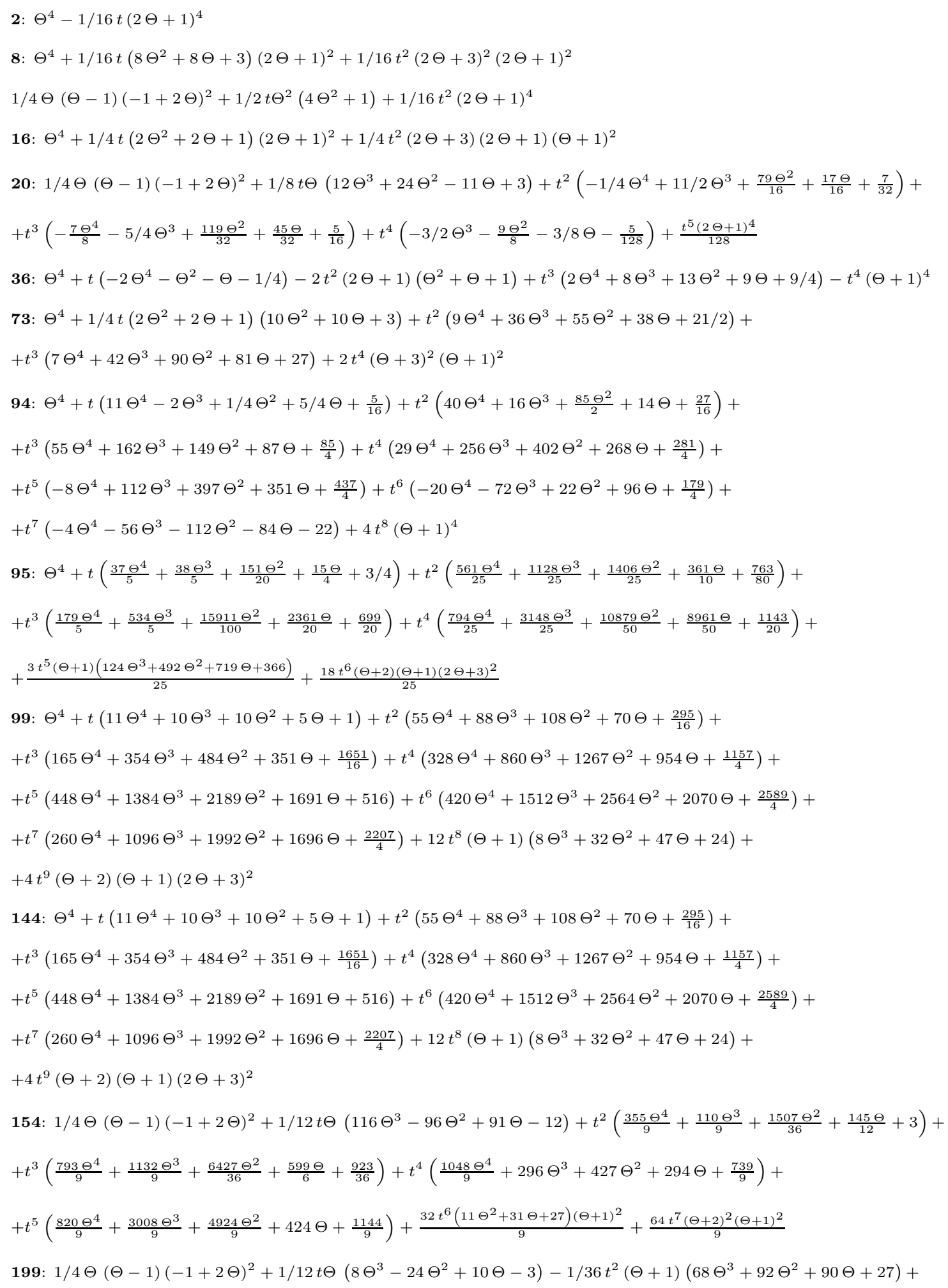




$$
\begin{aligned}
& +t^{3}\left(-4 / 3 \Theta^{4}-4 / 3 \Theta^{3}-\frac{11 \Theta^{2}}{9}-\frac{13 \Theta}{36}+1 / 36\right)+t^{4}\left(\frac{7 \Theta^{4}}{9}+6 \Theta^{3}+\frac{49 \Theta^{2}}{4}+21 / 2 \Theta+\frac{115}{36}\right)+ \\
& +t^{5}\left(2 / 3 \Theta^{4}+10 / 3 \Theta^{3}+11 / 2 \Theta^{2}+\frac{23 \Theta}{6}+\frac{35}{36}\right)+1 / 9 t^{6}(\Theta+1)^{4} \\
& \text { 242: } \Theta^{4}+t\left(5 \Theta^{4}+10 \Theta^{3}+10 \Theta^{2}+5 \Theta+1\right)+t^{2}\left(9 \Theta^{2}+18 \Theta+14\right)(\Theta+1)^{2}+t^{3}(\Theta+2)(\Theta+1)\left(7 \Theta^{2}+21 \Theta+18\right)+ \\
& +2 t^{4}(\Theta+3)(\Theta+1)(\Theta+2)^{2} \\
& \text { 246: } 1 / 4 \Theta(\Theta-1)(-1+2 \Theta)^{2}+1 / 4 t \Theta^{2}\left(20 \Theta^{2}+11\right)+t^{2}\left(9 \Theta^{4}+18 \Theta^{3}+22 \Theta^{2}+13 \Theta+\frac{51}{16}\right)+ \\
& +1 / 16 t^{3}\left(4 \Theta^{2}+8 \Theta+7\right)\left(28 \Theta^{2}+56 \Theta+29\right)+1 / 8 t^{4}(2 \Theta+3)^{4} \\
& \text { 249: } \Theta^{2}(\Theta-1)^{2}+t \Theta^{2}\left(4 \Theta^{2}+1\right)+t^{2}\left(5 \Theta^{4}+10 \Theta^{3}+10 \Theta^{2}+5 \Theta+1\right)+2 t^{3}(\Theta+1)^{4} \\
& \text { 251: } 1 / 4 \Theta(\Theta-1)(-1+2 \Theta)^{2}+1 / 14 t \Theta\left(232 \Theta^{3}-360 \Theta^{2}+261 \Theta-45\right)+t^{2}\left(\frac{5989 \Theta^{4}}{49}-\frac{6526 \Theta^{3}}{49}+\frac{12673 \Theta^{2}}{98}-\frac{1321 \Theta}{98}+\frac{69}{56}\right)+ \\
& +t^{3}\left(\frac{25873 \Theta^{4}}{49}-\frac{2336 \Theta^{3}}{7}+\frac{54315 \Theta^{2}}{98}+\frac{115 \Theta}{49}+\frac{11325}{784}\right)+t^{4}\left(\frac{72358 \Theta^{4}}{49}-\frac{14880 \Theta^{3}}{49}+\frac{44351 \Theta^{2}}{28}+\frac{4785 \Theta}{28}+\frac{45387}{784}\right)+ \\
& +t^{5}\left(\frac{136135 \Theta^{4}}{49}+\frac{2768 \Theta^{3}}{7}+\frac{141170 \Theta^{2}}{49}+\frac{33581 \Theta}{98}+\frac{19353}{784}\right)+t^{6}\left(\frac{171792 \Theta^{4}}{49}+\frac{52596 \Theta^{3}}{49}+\frac{118302 \Theta^{2}}{49}-\frac{42657 \Theta}{49}-\frac{4131}{7}\right)+ \\
& +t^{7}\left(\frac{19472 \Theta^{4}}{7}-\frac{1128 \Theta^{3}}{7}-\frac{16601 \Theta^{2}}{7}-\frac{267159 \Theta}{49}-\frac{470223}{196}\right)+t^{8}\left(\frac{49752 \Theta^{4}}{49}-\frac{169128 \Theta^{3}}{49}-\frac{492384 \Theta^{2}}{49}-\frac{593742 \Theta}{49}-\frac{471897}{98}\right)+ \\
& +t^{9}\left(-\frac{19296 \Theta^{4}}{49}-\frac{283680 \Theta^{3}}{49}-\frac{100188 \Theta^{2}}{7}-\frac{747468 \Theta}{49}-\frac{284463}{49}\right)+t^{10}\left(-\frac{34656 \Theta^{4}}{49}-\frac{34560 \Theta^{3}}{7}-\frac{563700 \Theta^{2}}{49}-\frac{574668 \Theta}{49}-\frac{213219}{49}\right)+ \\
& +t^{11}\left(-\frac{2736 \Theta^{4}}{7}-\frac{117792 \Theta^{3}}{49}-\frac{267408 \Theta^{2}}{49}-\frac{265968 \Theta}{49}-\frac{96687}{49}\right)-\frac{432 t^{12}(\Theta+1)\left(12 \Theta^{3}+60 \Theta^{2}+101 \Theta+56\right)}{49}-\frac{144 t^{13}(\Theta+2)(\Theta+1)(2 \Theta+3)^{2}}{49} \\
& \text { 253: } 1 / 4 \Theta(\Theta-1)(-1+2 \Theta)^{2}+1 / 40 t \Theta\left(492 \Theta^{3}-456 \Theta^{2}+379 \Theta-57\right)+t^{2}\left(\frac{6731 \Theta^{4}}{100}+\frac{83 \Theta^{3}}{50}+\frac{25109 \Theta^{2}}{400}+\frac{4989 \Theta}{400}+\frac{553}{160}\right)+ \\
& +t^{3}\left(\frac{43629 \Theta^{4}}{200}+\frac{18723 \Theta^{3}}{100}+\frac{264889 \Theta^{2}}{800}+\frac{124409 \Theta}{800}+\frac{31623}{800}\right)+t^{4}\left(\frac{47001 \Theta^{4}}{100}+\frac{37083 \Theta^{3}}{50}+\frac{880637 \Theta^{2}}{800}+\frac{268839 \Theta}{400}+\frac{578301}{3200}\right)+ \\
& +t^{5}\left(\frac{28631 \Theta^{4}}{40}+\frac{156673 \Theta^{3}}{100}+\frac{1863431 \Theta^{2}}{800}+\frac{1285873 \Theta}{800}+\frac{90707}{200}\right)+t^{6}\left(\frac{159683 \Theta^{4}}{200}+\frac{107837 \Theta^{3}}{50}+\frac{2649937 \Theta^{2}}{800}+\frac{975887 \Theta}{400}+\frac{568541}{800}\right)+ \\
& +t^{7}\left(\frac{66527 \Theta^{4}}{100}+\frac{52122 \Theta^{3}}{25}+\frac{66313 \Theta^{2}}{20}+\frac{505161 \Theta}{200}+\frac{149699}{200}\right)+t^{8}\left(\frac{83369 \Theta^{4}}{200}+\frac{36614 \Theta^{3}}{25}+\frac{482423 \Theta^{2}}{200}+\frac{187197 \Theta}{100}+\frac{22373}{40}\right)+ \\
& +t^{9}\left(\frac{19509 \Theta^{4}}{100}+\frac{37767 \Theta^{3}}{50}+\frac{129321 \Theta^{2}}{100}+\frac{51153 \Theta}{50}+\frac{7713}{25}\right)+t^{10}\left(\frac{333 \Theta^{4}}{5}+\frac{7068 \Theta^{3}}{25}+\frac{25289 \Theta^{2}}{50}+\frac{10299 \Theta}{25}+\frac{6327}{50}\right)+ \\
& +t^{11}\left(\frac{394 \Theta^{4}}{25}+\frac{1844 \Theta^{3}}{25}+\frac{3464 \Theta^{2}}{25}+\frac{2926 \Theta}{25}+\frac{922}{25}\right)+\frac{2}{25} t^{12}\left(29 \Theta^{2}+94 \Theta+86\right)(\Theta+1)^{2}+\frac{4 t^{13}(\Theta+2)^{2}(\Theta+1)^{2}}{25} \\
& \text { 254: } \Theta^{4}+t\left(10 \Theta^{4}+14 \Theta^{3}+\frac{25}{2} \Theta^{2}+11 / 2 \Theta+\frac{17}{16}\right)+t^{2}\left(62 \Theta^{4}+74 \Theta^{3}+104 \Theta^{2}+\frac{171 \Theta}{2}+\frac{399}{16}\right)+ \\
& +t^{3}\left(293 \Theta^{4}+276 \Theta^{3}+\frac{271 \Theta^{2}}{2}+228 \Theta+\frac{331}{4}\right)+t^{4}\left(1039 \Theta^{4}+944 \Theta^{3}+338 \Theta^{2}-47 \Theta-\frac{1829}{16}\right)+ \\
& +t^{5}\left(2884 \Theta^{4}+2098 \Theta^{3}+\frac{5937 \Theta^{2}}{2}+\frac{275 \Theta}{2}-\frac{4281}{8}\right)+t^{6}\left(6348 \Theta^{4}+4278 \Theta^{3}+9109 \Theta^{2}+\frac{6591 \Theta}{2}+72\right)+ \\
& +t^{7}\left(10465 \Theta^{4}+10988 \Theta^{3}+\frac{36131 \Theta^{2}}{2}+10011 \Theta+\frac{37033}{16}\right)+t^{8}\left(12062 \Theta^{4}+21584 \Theta^{3}+30237 \Theta^{2}+19844 \Theta+\frac{44823}{8}\right)+ \\
& +t^{9}\left(9196 \Theta^{4}+25056 \Theta^{3}+37002 \Theta^{2}+27144 \Theta+\frac{32891}{4}\right)+t^{10}\left(4360 \Theta^{4}+16352 \Theta^{3}+27484 \Theta^{2}+22392 \Theta+\frac{14529}{2}\right)+ \\
& +t^{11}\left(1152 \Theta^{4}+5568 \Theta^{3}+10816 \Theta^{2}+9776 \Theta+3416\right)+8 t^{12}(2 \Theta+3)^{4} \\
& \text { 255: } \Theta^{4}+1 / 4 t \Theta\left(4 \Theta^{3}-10 \Theta^{2}-6 \Theta-1\right)+t^{2}\left(-\frac{23 \Theta^{4}}{16}-\frac{31 \Theta^{3}}{8}-\frac{25 \Theta^{2}}{8}-\frac{57 \Theta}{16}-\frac{89}{64}\right)+ \\
& +t^{3}\left(-\frac{21 \Theta^{4}}{32}-\frac{21 \Theta^{3}}{8}-\frac{83 \Theta^{2}}{16}-\frac{33 \Theta}{32}+\frac{51}{128}\right)+t^{4}\left(\frac{59 \Theta^{4}}{64}+\frac{41 \Theta^{3}}{32}+\frac{279 \Theta^{2}}{32}+\frac{923 \Theta}{128}+\frac{2567}{1024}\right)+ \\
& +t^{5}\left(-\frac{11 \Theta^{4}}{32}+\frac{91 \Theta^{3}}{32}+\frac{3477 \Theta^{2}}{512}+\frac{1907 \Theta}{256}+\frac{1741}{512}\right)+t^{6}\left(-\frac{27 \Theta^{4}}{128}-\frac{75 \Theta^{3}}{64}-\frac{9 \Theta^{2}}{16}-\frac{657 \Theta}{512}-\frac{697}{1024}\right)+ \\
& +t^{7}\left(\frac{57 \Theta^{4}}{256}+\frac{39 \Theta^{3}}{128}-\frac{3085 \Theta^{2}}{2048}-\frac{2795 \Theta}{1024}-\frac{3089}{2048}\right)+t^{8}\left(-\frac{11 \Theta^{4}}{512}+\frac{113 \Theta^{3}}{256}+\frac{601 \Theta^{2}}{2048}+\frac{9 \Theta}{256}-\frac{1027}{8192}\right)+ \\
& +t^{9}\left(-\frac{13 \Theta^{4}}{512}-\frac{57 \Theta^{3}}{256}-\frac{2581 \Theta^{2}}{8192}-\frac{717 \Theta}{4096}-\frac{73}{8192}\right)+t^{10}\left(\frac{61 \Theta^{4}}{4096}-\frac{11 \Theta^{3}}{2048}-\frac{359 \Theta^{2}}{4096}-\frac{1005 \Theta}{8192}-\frac{205}{4096}\right)+
\end{aligned}
$$




$$
\begin{aligned}
& +t^{11}\left(-\frac{5 \Theta^{4}}{8192}+\frac{5 \Theta^{3}}{1024}+\frac{725 \Theta^{2}}{32768}+\frac{475 \Theta}{16384}+\frac{205}{16384}\right)-\frac{25 t^{12}(2 \Theta+3)^{4}}{262144} \\
& \text { 256: } 1 / 16(2 \Theta+1)^{4}+t\left(-21 / 2 \Theta^{4}-12 \Theta^{3}+3 \Theta^{2}+15 / 2 \Theta+\frac{35}{16}\right)+t^{2}\left(\frac{61 \Theta^{4}}{2}+15 \Theta^{3}-\frac{21 \Theta^{2}}{4}+3 \Theta+\frac{95}{32}\right)+ \\
& +t^{3}\left(-\frac{121 \Theta^{4}}{8}-22 \Theta^{3}-\frac{51 \Theta^{2}}{4}-\frac{23 \Theta}{8}+\frac{23}{64}\right)-3 / 16 t^{4}(\Theta+1)\left(44 \Theta^{2}+88 \Theta+61\right)+ \\
& +t^{5}\left(\frac{121 \Theta^{4}}{32}+\frac{99 \Theta^{3}}{4}+\frac{975 \Theta^{2}}{16}+\frac{2145 \Theta}{32}+\frac{7097}{256}\right)+t^{6}\left(-\frac{61 \Theta^{4}}{32}-\frac{229 \Theta^{3}}{16}-\frac{2547 \Theta^{2}}{64}-\frac{193 \Theta}{4}-\frac{11007}{512}\right)+ \\
& +t^{7}\left(\frac{21 \Theta^{4}}{128}+\frac{9 \Theta^{3}}{8}+\frac{177 \Theta^{2}}{64}+\frac{375 \Theta}{128}+\frac{1165}{1024}\right)-\frac{t^{8}(2 \Theta+3)^{4}}{4096} \\
& \text { 257: } \Theta^{2}(\Theta-1)^{2}-1 / 4 t \Theta^{2}\left(13 \Theta^{2}-12 \Theta+9\right)+t^{2}\left(\frac{33 \Theta^{4}}{8}+\frac{15 \Theta^{3}}{4}+\frac{17 \Theta^{2}}{4}+\frac{13 \Theta}{8}+3 / 8\right)+ \\
& t^{3}\left(-\frac{77 \Theta^{4}}{32}-\frac{43 \Theta^{3}}{4}-\frac{677 \Theta^{2}}{64}-\frac{119 \Theta}{16}-\frac{33}{16}\right)+t^{4}\left(\frac{85 \Theta^{4}}{256}+\frac{1059 \Theta^{3}}{128}+\frac{3735 \Theta^{2}}{256}+\frac{753 \Theta}{64}+\frac{499}{128}\right)+ \\
& +t^{5}\left(\frac{423 \Theta^{4}}{1024}-\frac{495 \Theta^{3}}{256}-\frac{9145 \Theta^{2}}{1024}-\frac{2317 \Theta}{256}-\frac{3715}{1024}\right)+t^{6}\left(-\frac{271 \Theta^{4}}{1024}-\frac{419 \Theta^{3}}{512}+\frac{1699 \Theta^{2}}{1024}+\frac{6125 \Theta}{2048}+\frac{3229}{2048}\right)+ \\
& +t^{7}\left(\frac{187 \Theta^{4}}{4096}+\frac{297 \Theta^{3}}{512}+\frac{9423 \Theta^{2}}{16384}+\frac{105 \Theta}{2048}-\frac{695}{4096}\right)+t^{8}\left(\frac{111 \Theta^{4}}{8192}-\frac{321 \Theta^{3}}{4096}-\frac{1859 \Theta^{2}}{8192}-\frac{1745 \Theta}{8192}-\frac{1055}{16384}\right)+ \\
& +t^{9}\left(-\frac{55 \Theta^{4}}{8192}-\frac{5 \Theta^{3}}{256}-\frac{1125 \Theta^{2}}{65536}-\frac{25 \Theta}{32768}+\frac{955}{262144}\right)+\frac{25 t^{10}(2 \Theta+3)^{4}}{524288} \\
& \text { 259: } 1 / 4 \Theta(\Theta-1)(-1+2 \Theta)^{2}+1 / 2 t \Theta\left(8 \Theta^{2}-4 \Theta+1\right)+t^{2}\left(-3 \Theta^{4}-2 \Theta^{3}-\frac{15 \Theta^{2}}{4}-\frac{17 \Theta}{4}-1\right)- \\
& -1 / 2 t^{3}(2 \Theta+1)\left(8 \Theta^{2}+8 \Theta+13\right)+t^{4}\left(3 \Theta^{4}+10 \Theta^{3}+\frac{63 \Theta^{2}}{4}+\frac{37 \Theta}{4}+3 / 2\right)+1 / 2 t^{5}(\Theta+1)\left(8 \Theta^{2}+20 \Theta+13\right)- \\
& -1 / 4 t^{6}(\Theta+2)(\Theta+1)(2 \Theta+3)^{2} \\
& \text { 262: } 1 / 4 \Theta(\Theta-1)(-1+2 \Theta)^{2}+1 / 28 t \Theta\left(164 \Theta^{3}-72 \Theta^{2}+111 \Theta-9\right)+t^{2}\left(\frac{807 \Theta^{4}}{49}+\frac{930 \Theta^{3}}{49}+\frac{2623 \Theta^{2}}{98}+\frac{1261 \Theta}{98}+\frac{177}{56}\right)+ \\
& +t^{3}\left(\frac{1417 \Theta^{4}}{49}+\frac{4012 \Theta^{3}}{49}+\frac{23965 \Theta^{2}}{196}+\frac{17571 \Theta}{196}+\frac{22095}{784}\right)+t^{4}\left(\frac{1670 \Theta^{4}}{49}+\frac{7806 \Theta^{3}}{49}+\frac{58725 \Theta^{2}}{196}+\frac{52533 \Theta}{196}+\frac{76995}{784}\right)+ \\
& +t^{5}\left(\frac{1308 \Theta^{4}}{49}+\frac{9096 \Theta^{3}}{49}+\frac{86145 \Theta^{2}}{196}+\frac{89409 \Theta}{196}+\frac{36555}{196}\right)+t^{6}\left(12 \Theta^{4}+\frac{930 \Theta^{3}}{7}+\frac{79011 \Theta^{2}}{196}+\frac{94125 \Theta}{196}+\frac{24207}{112}\right)+ \\
& +t^{7}\left(\frac{12 \Theta^{4}}{49}+\frac{2340 \Theta^{3}}{49}+\frac{10656 \Theta^{2}}{49}+\frac{29817 \Theta}{98}+\frac{2115}{14}\right)+t^{8}\left(-\frac{177 \Theta^{4}}{49}-\frac{282 \Theta^{3}}{49}+\frac{9369 \Theta^{2}}{196}+\frac{19419 \Theta}{196}+\frac{22899}{392}\right)+ \\
& +t^{9}\left(-\frac{115 \Theta^{4}}{49}-\frac{724 \Theta^{3}}{49}-\frac{1495 \Theta^{2}}{98}+\frac{151 \Theta}{49}+\frac{3639}{392}\right)+t^{10}\left(-\frac{26 \Theta^{4}}{49}-\frac{306 \Theta^{3}}{49}-\frac{1275 \Theta^{2}}{98}-\frac{999 \Theta}{98}-\frac{1683}{784}\right)+ \\
& +t^{11}\left(\frac{6 \Theta^{4}}{49}-\frac{24 \Theta^{3}}{49}-\frac{401 \Theta^{2}}{196}-\frac{461 \Theta}{196}-\frac{663}{784}\right)+t^{12}\left(\frac{5 \Theta^{4}}{49}+\frac{20 \Theta^{3}}{49}+\frac{32 \Theta^{2}}{49}+\frac{24 \Theta}{49}+\frac{57}{392}\right)+\frac{t^{13}(2 \Theta+3)^{4}}{784} \\
& \text { 265: } 1 / 4 \Theta^{2}(-1+2 \Theta)(2 \Theta+1)-1 / 32 t(2 \Theta+1)\left(32 \Theta^{3}+16 \Theta^{2}+22 \Theta+7\right)+t^{2}\left(\frac{25 \Theta^{4}}{16}+\frac{13 \Theta^{3}}{4}+\frac{129 \Theta^{2}}{32}+\frac{41 \Theta}{16}+\frac{165}{256}\right)+ \\
& +t^{3}\left(-\frac{19 \Theta^{4}}{32}-\frac{31 \Theta^{3}}{16}-\frac{189 \Theta^{2}}{64}-\frac{143 \Theta}{64}-\frac{347}{512}\right)+t^{4}\left(\frac{7 \Theta^{4}}{64}+1 / 2 \Theta^{3}+\frac{119 \Theta^{2}}{128}+\frac{13 \Theta}{16}+\frac{283}{1024}\right)-\frac{t^{5}(2 \Theta+3)^{4}}{2048} \\
& \text { 268: } \Theta^{4}-t \Theta\left(4 \Theta^{3}-10 \Theta^{2}-6 \Theta-1\right)+t^{2}\left(-23 \Theta^{4}-62 \Theta^{3}-50 \Theta^{2}-57 \Theta-\frac{89}{4}\right)+t^{3}\left(42 \Theta^{4}+168 \Theta^{3}+332 \Theta^{2}+66 \Theta-\frac{51}{2}\right)+ \\
& +t^{4}\left(236 \Theta^{4}+328 \Theta^{3}+2232 \Theta^{2}+1846 \Theta+\frac{2567}{4}\right)+t^{5}\left(352 \Theta^{4}-2912 \Theta^{3}-6954 \Theta^{2}-7628 \Theta-3482\right)+ \\
& +t^{6}\left(-864 \Theta^{4}-4800 \Theta^{3}-2304 \Theta^{2}-5256 \Theta-2788\right)+t^{7}\left(-3648 \Theta^{4}-4992 \Theta^{3}+24680 \Theta^{2}+44720 \Theta+24712\right)+ \\
& +t^{8}\left(-1408 \Theta^{4}+28928 \Theta^{3}+19232 \Theta^{2}+2304 \Theta-8216\right)+t^{9}\left(6656 \Theta^{4}+58368 \Theta^{3}+82592 \Theta^{2}+45888 \Theta+2336\right)+ \\
& +t^{10}\left(15616 \Theta^{4}-5632 \Theta^{3}-91904 \Theta^{2}-128640 \Theta-52480\right)+t^{11}\left(2560 \Theta^{4}-20480 \Theta^{3}-92800 \Theta^{2}-121600 \Theta-52480\right)- \\
& -1600 t^{12}(2 \Theta+3)^{4} \\
& \text { 274: } 1 / 4 \Theta(\Theta-1)(-1+2 \Theta)^{2}+1 / 40 t \Theta\left(332 \Theta^{3}-216 \Theta^{2}+219 \Theta-27\right)+t^{2}\left(\frac{2871 \Theta^{4}}{100}+\frac{1413 \Theta^{3}}{50}+\frac{12849 \Theta^{2}}{400}+\frac{5199 \Theta}{400}+\frac{95}{32}\right)+ \\
& +t^{3}\left(\frac{10169 \Theta^{4}}{200}+\frac{17737 \Theta^{3}}{100}+\frac{152361 \Theta^{2}}{800}+\frac{92291 \Theta}{800}+\frac{999}{32}\right)+t^{4}\left(\frac{1873 \Theta^{4}}{50}+\frac{40143 \Theta^{3}}{100}+\frac{506483 \Theta^{2}}{800}+\frac{352509 \Theta}{800}+\frac{89327}{640}\right)+ \\
& +t^{5}\left(-\frac{2871 \Theta^{4}}{100}+\frac{42237 \Theta^{3}}{100}+\frac{942333 \Theta^{2}}{800}+\frac{752883 \Theta}{800}+\frac{545069}{1600}\right)+t^{6}\left(-\frac{19521 \Theta^{4}}{200}+\frac{4491 \Theta^{3}}{100}+\frac{962217 \Theta^{2}}{800}+\frac{185391 \Theta}{160}+\frac{1549843}{3200}\right)+
\end{aligned}
$$




$$
\begin{aligned}
& +t^{7}\left(-\frac{19521 \Theta^{4}}{200}-\frac{43533 \Theta^{3}}{100}+\frac{385929 \Theta^{2}}{800}+\frac{577359 \Theta}{800}+\frac{1234843}{3200}\right)+t^{8}\left(-\frac{2871 \Theta^{4}}{100}-\frac{53721 \Theta^{3}}{100}-\frac{209163 \Theta^{2}}{800}+\frac{26223 \Theta}{800}+\frac{202241}{1600}\right)+ \\
& +t^{9}\left(\frac{1873 \Theta^{4}}{50}-\frac{25159 \Theta^{3}}{100}-\frac{277141 \Theta^{2}}{800}-\frac{183103 \Theta}{800}-\frac{102173}{3200}\right)+t^{10}\left(\frac{10169 \Theta^{4}}{200}+\frac{2601 \Theta^{3}}{100}-\frac{29271 \Theta^{2}}{800}-\frac{50553 \Theta}{800}-\frac{647}{32}\right)+ \\
& +t^{11}\left(\frac{2871 \Theta^{4}}{100}+\frac{4329 \Theta^{3}}{50}+\frac{47841 \Theta^{2}}{400}+\frac{32523 \Theta}{400}+\frac{3607}{160}\right)+1 / 40 t^{12}(\Theta+1)\left(332 \Theta^{3}+1212 \Theta^{2}+1647 \Theta+794\right)+ \\
& +1 / 4 t^{13}(\Theta+2)(\Theta+1)(2 \Theta+3)^{2}
\end{aligned}
$$

\section{REFERENCES}

[1] S. Cynk, B. Kocel-Cynk, Classification of double octic Calabi-Yau threefolds, Commun. Contemp. Math. (to appear), arXiv:1612.04364 [math.AG].

[2] S. Cynk, D. van Straten, Periods of rigid double octic CalabiYau threefolds, Ann. Polon. Math. 123, 2019, $243-258$.

[3] S. Cynk, D. van Straten, Picard-Fuchs operators for octic arrangements II, (The MUM cases), in preparation.

[4] C. van Enckevort, D. van Straten, Monodromy calculations of fourth order equations of Calabi-Yau type. Mirror symmetry. V, 539559, AMS/IP Stud. Adv. Math., 38, Amer. Math. Soc., Providence, RI, 2006.

[5] F. Gouêva, N. Yui, Rigid Calabi-Yau threefolds over $\mathbb{Q}$ are modular. Expositiones Mathematicae 29, 2011, $142-149$.

[6] M. Gross, D. Huybrechts, D. Joyce, Calabi-Yau Manifolds and Related Geometries. Springer-Verlag Berlin Heidelberg New York, 2003.

[7] E. Ince, Ordinary Differential Equations, Longmans, Green and Co. Ltd., New York, Toronto, Calcutta, Madras (1927).

[8] C. Meyer, Modular Calabi-Yau threefolds. Fields Institute Monographs, 22. American Mathematical Society, Providence, RI, 2005.

[9] H. Ruddat, B. Siebert, Period integrals from wall structures via tropical cycles, canonical coordinates in mirror symmetry and analyticity of toric degenerations. arXiv:1907.03794 [math.AG]

[10] D. van Straten, Calabi-Yau Operators, in: Uniformization, Riemann-Hilbert Correspondence, Calabi-Yau Manifolds and Picard-Fuchs Equations, 401-452, Advanced Lectures in Mathematics 42, International Press, Boston (2018).

[11] J. Werner, Kleine Auflösungen spezieller dreidimensionaler Varietäten, Bonner Math. Schriften 186 (1987).

Jagiellonian University, ul. Łojasiewicza 6, 30-348 Kraków, Poland 